REVASCAT and SWIFT PRIME, demonstrated a reduction in the rate for sICH of the THERAPY cohort to as low as $0.0 \%$ for the intervention arm (Table 1).

Conclusion Under the trial definition, any apparent extravascular blood in the brain or cranium with an increase of 4 or more on the NIHSS, associated or not, is considered a sICH; as a result, the inclusion of hemorrhagic infarctions (HI1 and HI2) in this definition resulted in an apparent relative increase in sICH rates. Subjecting the THERAPY cohort to other definitions yielded notable variance in complication rates. As there is no current standardization for the quantification of sICH, further analysis and data are needed to identify and standardize a succinct and relevant definition.

Disclosures R. von Kummer: 2; C; Penumbra, Inc. D. Frei: 3; C; Penumbra, Inc. A. Yoo: 1; C; National Institute of Health, Penumbra, Inc., Remedy Pharmaceuticals. O. Zaidat: 6; C; Penumbra, Inc. P. Khatri: 1; C; Penumbra, Inc. R. Gupta: 6; C; Penumbra, Inc. D. Lopes: 6; C; Penumbra, Inc. H. Shownkeen: None. D. Meyer: 5; C; Penumbra, Inc. H. Buell: 5; C; Penumbra, Inc. V. Bach: 5; C; Penumbra, Inc. S. Kuo: 5; C; Penumbra, Inc. A. Bose: 4; C; Penumbra, Inc. 5; C; Penumbra, Inc. S. Sit: 4; C; Penumbra, Inc. 5; C; Penumbra, Inc. J. Mocco: 1; C; Penumbra, Inc.

\section{0-012 EMERGENT ENDOVASCULAR MANAGEMENT OF LONG- SEGMENT CAROTID ARTERY DISSECTIONS IN ACUTE ISCHEMIC STROKE INTERVENTION WITH MULTIPLE TANDEM STENTS}

${ }^{1} \mathrm{~S}$ Ansari, ${ }^{2} \mathrm{~A}$ Kuhn, ${ }^{3} \mathrm{~A}$ Honarmand, ${ }^{4} \mathrm{~S}$ Hou, ${ }^{5} \mathrm{M}$ Khan, ${ }^{2} \mathrm{~J}$ Chueh, ${ }^{2} \mathrm{I}$ van der Bom, ${ }^{1} \mathrm{M}$ Hurley, ${ }^{1} \mathrm{~A}$ Shaibani, ${ }^{2} \mathrm{M}$ Gounis, ${ }^{1} \mathrm{M}$ Potts, ${ }^{1} \mathrm{~B}$ Jahromi, ${ }^{2} \mathrm{~A}$ Wakhloo, ${ }^{2} \mathrm{~A}$ Puri. ${ }^{1}$ Radiology and Neurological Surgery, Northwestern University Feinberg School of Medicine, Chicago, IL; ${ }^{2}$ Radiology, University of Massachusetts, Worcester, MA; ${ }^{3}$ Radiology, Northwestern University Feinberg School of Medicine, Chicago, IL; ${ }^{4}$ Stroke and Neurovascular center of Central California, Santa Barbara, CA; ${ }^{5}$ Neurology, University of Massachusetts, Worcester, MA

\subsection{6/neurintsurg-2016-012589.12}

Background and purpose Cervical dissections are a significant cause of acute ischemic stroke, especially in young and middle aged adults. Although medical management of cervical dissections is standard treatment, emergent endovascular treatment may become necessary in the presence of acute intracranial large vessel occlusions, flow limiting and long segment lesions with impending occlusion, and/or ischemia at risk for cerebral infarction. We report our experience with the endovascular reconstruction of long segment carotid dissections using multiple tandem stents in acute ischemic stroke intervention.

Materials and methods We retrospectively studied patients with carotid artery dissections requiring stent reconstruction at our institutions between January 2011 and January 2015, presenting with acute ( $<12$ hours), severe ischemic stroke symptoms (NIHSS $>4$ ), and requiring carotid reconstruction with multiple tandem stents ( $\geq 3$ stents). We analyzed patients' demographics, vascular risk factors, presentations, imaging and angiographic findings, technical efficacy and safety, and clinical outcomes.

Results We identified 15 patients presenting with acute ischemic stroke that underwent endovascular stent reconstruction of carotid dissections for vessel and/or ischemic tissue salvage. Mean patient age was 51.5 years with a mean presenting NIHSS of 15 and discharge NIHSS of 6. All carotid dissections presented with $>70 \%$ stenosis and severe flow limitation, nearly all 14/15 involving the distal cervical segment of the ICA with a minimum length of $3.5 \mathrm{~cm}$. Technical success of carotid stent reconstruction was achieved in all patients $(100 \%)$ with no significant residual stenosis or flow limitation. Nine patients $(60 \%)$ harbored simultaneous intracranial occlusions, and 6 patients (40\%) required IA thrombolysis/thrombectomy achieving TICI 2 b-3 reperfusion in all 15 patients. There were no symptomatic intracranial hemorrhages. Procedural complications were limited to distal thromboemboli and multifocal infarcts in a single patient due to suspected in-stent thrombus, resulting in 7\% procedural morbidity and $0 \%$ mortality. Follow-up 3-6 month angiographic and ultrasound imaging evaluations confirmed normalization of carotid artery caliber and stent patency in 12/14 patients, with 2 cases of only mild persistent vessel irregularity and $<20 \%$ in-stent stenosis. On clinical follow-up, 9/15 (60\%) of patients achieved mRS $\leq 2$ at 90 days, with no interval recurrent TIAs or strokes.

Conclusion Tandem stent reconstruction for the treatment of long segment and flow limiting carotid dissections is technically safe and effective with favorable clinical outcomes in acute ischemic stroke intervention, allowing for successful thrombectomy, vessel salvage, restoration of cerebral perfusion, and prevention of recurrent thromboembolic stroke.

Disclosures S. Ansari: None. A. Kuhn: None. A. Honarmand: None. S. Hou: None. M. Khan: None. J. Chueh: None. I. van der Bom: None. M. Hurley: None. A. Shaibani: None. M. Gounis: None. M. Potts: None. B. Jahromi: None. A. Wakhloo: None. A. Puri: None.

\section{0-013 HYPERTENSION DECREASES THE NUMBER AND DURATION OF COLLATERAL OPENINGS DURING MIDDLE CEREBRAL ARTERY OCCLUSION (MCAO) AND IMPAIRS REPERFUSION}

M Cipolla, J Sweet, K Chan. Neurological Sciences, University of Vermont, Burlington, VT

\subsection{6/neurintsurg-2016-012589.13}

Introduction Hypertension is associated with large ischemic cores and little penumbral tissue that is thought to contribute to poor outcome from ischemic stroke. Pial collaterals (leptomeningeal anastomoses; LMA) are thought to perfuse the penumbra during MCAO by promoting retrograde flow from the anterior cerebral artery (ACA) to the MCA territory. We recently demonstrated that pial collaterals are vasoconstricted in spontaneously hypertensive rats (SHR) that likely contributes to poor collateral flow and increased infarction. Here, we developed methodology to measure collateral openings in SHR $(\mathrm{n}=6)$ compared to normotensive Wistar $(\mathrm{n}=6)$ rats during MCAO. We hypothesized that SHR have fewer collateral openings and/or shorter duration openings during MCAO, and impaired reperfusion.

Materials and methods Dual laser Doppler probes were used to simultaneously measure changes in relative cerebral blood flow (rCBF) in the MCA (probe 1: Bregma -2, lateral +4) and ACA collateral (probe 2: Bregma +2 , lateral +3 ) vascular territories during 2 hours of MCAO by filament occlusion (Figure 1). Collateral openings were defined as increased collateral flow that did not correspond to changes in blood pressure. The number and duration of collateral openings were quantified off rCBF tracings blinded to group. Animals were 
mechanically ventilated during MCAO to maintain blood gases within physiologic ranges and body temperature at $37^{\circ} \mathrm{C}$. Chloral hydrate anesthesia was used. Data are presented as mean \pm SEM.

Results The overall drop in CBF during ischemia was similar between Wistar and SHR $(-73 \pm 1 \%$ and $-78 \pm 4 \%$; $\mathrm{p}>0.05)$. The average number collateral openings during 2 hours of ischemia was decreased in SHR compared to Wistar $(0.8 \pm 0.6$ vs. $2.2 \pm 0.3 ; p=0.06)$, as was the total duration of collateral opening $(4 \pm 4$ vs. $26 \pm 8 \mathrm{~min}$; p $<0.05)$. After 2 hours of reperfusion, $\mathrm{rCBF}$ was decreased in the MCA territory in both SHR and Wistar compared to baseline $(-43 \pm 6 \%$ and $-24 \pm 4 \% ; \mathrm{p}<0.05)$ demonstrating incomplete microcirculatory perfusion; however, $\mathrm{rCBF}$ in the collateral perfusion territory was decreased in SHR and increased in Wistar (although variable) compared to baseline $(-26 \pm 7 \%$ vs. $70 \pm 63 \%)$.

Conclusions These results demonstrate that collateral flow during ischemia, and reperfusion, was impaired in hypertension likely due to vasoconstriction of LMAs. Understanding the function of pial collaterals under conditions such as hypertension, that is associated with little penumbral tissue, may provide targeted therapies to prevent collateral failure and extend the time window for treatment in these patients.

\section{Dual laser Doppler probe placement for core and collateral flow measurement on a rat brain}

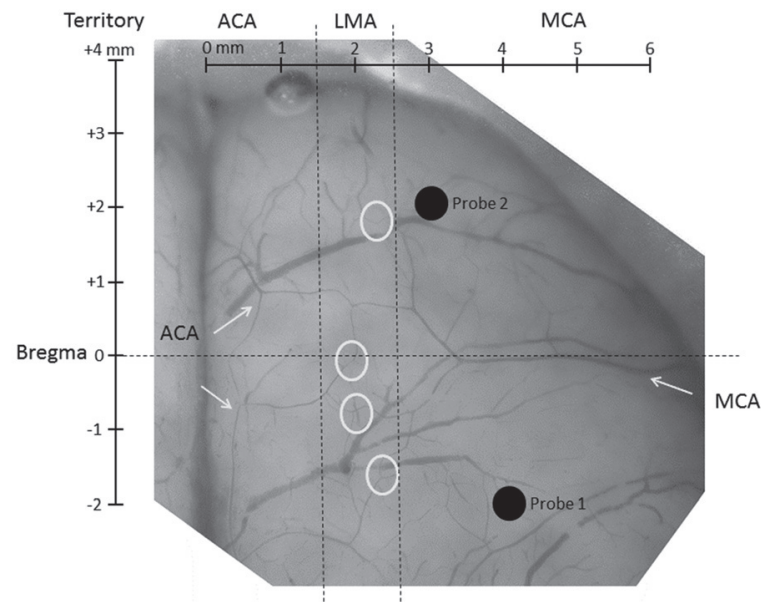

Abstract 0-013 Figure 1

Disclosures M. Cipolla: 1; C; NIH grants. J. Sweet: None. K. Chan: None.

\section{0-014 VISION OUTCOMES FOR PARACLINOID ANEURYSMS TREATED WITH FLOW DIVERTERS}

M Silva, A See, H Dasenbrock, P Khandelwal, N Patel, M Aziz-Sultan. Department of Neurosurgery, Brigham and Women's Hospital, Boston, MA

\subsection{6/neurintsurg-2016-012589.14}

Introduction Aneurysms of the paraclinoid region of the internal carotid artery (ICA) and the interventions used to treat them often result in visual impairment. Because of the prox-

\begin{tabular}{lllll} 
Abstract 0-014 Table 1 & & & \\
\hline Treatment & Improved & Unchanged & Deteriorated & $\begin{array}{l}\text { New } \\
\text { deficit }\end{array}$ \\
\hline Coil $(n=534)$ & & & & $9.2 \%$ \\
Clip ( $n=638)$ & $41.3 \%$ & $38.0 \%$ & $14.1 \%$ & $2.8 \%$ \\
Flow Diversion ( $=275)$ & $53.6 \%$ & $19.6 \%$ & $23.2 \%$ & $4.0 \%$ \\
Flow Diversion at BWH & $72.6 \%$ & $25.5 \%$ & $2.0 \%$ & $7.7 \%$ \\
( $n=23$ ) & $80 \%$ & $20 \%$ & $0 \%$ & \\
\hline
\end{tabular}

imity of these aneurysms to the optic nerve and the ophthalmic artery, between $16 \%$ and $53 \%$ of patients present with visual deficits. While flow diversion is increasingly used throughout endovascular neurosurgery, few studies have reported the visual outcomes of treating paraclinoid aneurysms with this technique or compared it to coiling or clipping.

Hypothesis In order to satisfy demand for data on the visual outcomes for this new endovascular treatment modality and inform the contemporary management of paraclinoid aneurysms, we performed a retrospective analysis of patients with paraclinoid aneurysms treated at our site with a Pipeline embolization device (PED).

Methods We compiled visual outcome data for 20 patients presenting with 26 paraclinoid aneurysms and treated at our site with flow diversion, 10 of whom (totaling 13 aneurysms) presented with visual impairment. We then reviewed vision outcomes in these patients at discharge and at 6 months, noting improved, unchanged, deteriorated, or newly impaired vision. We also performed an extensive literature review to compile data (1447 cases from 21 sites) on vision outcomes for paraclinoid aneurysms treated with clips (534 cases), coils (638), or flow diverters (275).

Results Of twenty patients with 26 paraclinoid aneurysms treated at our site with a PED, ten patients (totaling 13 aneurysms with an average diameter of $7.1 \mathrm{~mm}$ ) presented with preoperative visual impairment. Visual deficits were improved in 8 patients (80\%) and unchanged in $2(20 \%)$ following treatment. No patients experienced sustained worsening of their existing visual impairment, however one patient acquired postoperative iatrogenic vision impairment (5\%). A literature review of paraclinoid aneurysm treatment revealed visual improvement rates of approximately $41 \%$ for clipping, $54 \%$ for coiling, and $73 \%$ for flow diversion, visual deterioration rates of approximately $14 \%, 23 \%$, and $2 \%$, and newly impaired vision rates of $9 \%, 3 \%$, and $4 \%$.

Conclusion Compared to clipping and coiling, flow diversion demonstrates a higher rate of visual improvement and a lower rate of visual decline in patients with visually symptomatic paraclinoid aneurysms, with comparable risk of iatrogenic vision impairment. We report strong visual outcomes in patients treated at our site for paraclinoid aneurysms with a PED, including small aneurysms. Our results suggest that this new treatment modality yields improved vision outcomes in patients with paraclinoid aneurysms compared to clipping and coiling.

Disclosures M. Silva: None. A. See: None. H. Dasenbrock: None. P. Khandelwal: None. N. Patel: None. M. Aziz-Sultan: 2; C; Covidien. 